\title{
Competitive endogenous RNA network reveals that miR-1343 play key regulatory roles in longissimus dorsi muscle between Saba and Yorkshire pigs
}

Wei Hu

Yunnan Agricultural University

Qiang Chen

Yunnan Agricultural University

Xiaoyi Wang

Yunnan Agricultural University

Xinxing Dong

Yunnan Agricultural University

Mingli Li

Yunnan Agricultural University

Guoxiang Lan

Yunnan Agricultural University

Dawei Yan

Yunnan Agricultural University

Yu Yang

Yunnan Agricultural University

Shaoxiong Lu ( $\nabla$ shxlu@263.net)

Yunnan Agricultural University https://orcid.org/0000-0002-7006-9770

\section{Research article}

Keywords: Saba pig, Yorkshire pig, Meat quality, Longissimus Dorsi, Transcriptome, Competitive endogenous RNA network

Posted Date: December 3rd, 2019

DOI: https://doi.org/10.21203/rs.2.18063/v1

License: (c) (1) This work is licensed under a Creative Commons Attribution 4.0 International License.

Read Full License 


\section{Abstract}

Background Pork quality is an important economic trait in pig industry, and increasing data shows that Chinese indigenous and Western commercial pig breeds have significant differences in pork quality. However, the molecular regulatory mechanism of pork quality formation is still unclear. The aim of this study was to explore the transcriptional regulation mechanism of pork quality formation of Chinese Saba pig (SS) and Western Yorkshire pig (YY) breeds. Results A total of 31 DEmiRNAs, 902 DEmRNAs, 47 DElncRNAs and 198 DEcircRNAs were identified in longissimus dorsi muscles between SSs and YYs. On the basis of differentially expressed RNAs, two ceRNA networks including IncRNA-miRNA-mRNA and circRNA-miRNA-mRNA were established, and two ceRNA networks showed that miR-1343 had the most links in two ceRNA networks, which demonstrated that miR-1343 might play key regulatory roles in longissimus dorsi muscle formation between SS and YY breeds. Conclusions The present study identified miR-1343 as key regulatory factor in pig longissimus dorsi muscle formation by ceRNA networks, which provides novel insights into molecular regulatory mechanism of pork quality formation between fat-type and lean-type pig breeds.

\section{Background}

Pork quality is one of the most important economic trait [1,2], and high-quality pork results in a better economic benefit. Improving pork quality has been one of the major breeding objectives in pig industry [1, 3]. China is a wide pig-feeding country, with more than 80 indigenous pig breeds accounting for more than $30 \%$ pig resources in the world (Pig Genetic Resources in China, 2015). Among the pig indigenous genetic resources in China, Saba pig (SS) breed is an excellent indigenous breed in Yunnan Province of China, with higher pork quality including higher intramuscular fat content, and lower drip loss and muscle fiber diameter compared to Western commercial pig breeds such as Yorkshire pig (YY) breed. However, growth is lower and fat rate is higher in SS breed compared to YY breed, which seriously restricts the utilization and development of SS breed in pig breeding industry. To better utilize the two pig breeds with different excellent characteristics and produce fast-growth crossbreed pig with higher pork quality, it is vital to reveal the molecular difference of pork quality formation between SS and YY breeds.

Pork quality is a complex trait with the major characteristics including moisture, firmness, drip loss, color, $\mathrm{pH}$ value and intramuscular fat content $[4,5]$, and is effected by environment factors such as feeding and important genetics background such as breeds [6]. Currently, a few genes including ryanodine receptor 1 (RYR1), insulin-like growth factor(IGF2), gamma 3 non-catalytic subunit (PRKAG3) have been reported to relate with pork quality [7-10], and many quantitative trait loci (QTLs) associated with pork quality were found (https://www.animalgenome.org/). Despite advances in pork quality, these candidate genes and QTLs are not well applied to pig breeding industry due to population specificity of candidate genes and greater size of most QTLs. Genome-wide association studies (GWAS) have been demonstrated as a powerful approach to study complex traits, and a large number of single nucleotide polymorphisms (SNPs) associated with some aspects of pork quality were identified using GWAS [3, 11, 12]. For example, Liu et al. conducted GWAS for pork quality traits using Western DLY (Duroc×(LandracexYorkshire)) 
commercial population and Chinese Erhualian population, and totally identified 37 SNPs on 14 chromosomes associated with drip loss, pH and pork color [3]. Again, Xiong et al. performed GWAS for pork quality traits for 316 Chinese Laiwu pigs, and 75 SNPs were identified to associate with pork quality [13]. These studies contribute to uncovering the genetic mechanism involved in pork quality, and provide help for pork quality improvement in pig industry. However, studies on the molecular regulatory mechanisms involved in pork quality are relatively rare compared to genetic mechanisms, and few advances were obtained.

With the rapid progression of sequencing technology in recent years, advances were produced in regulatory mechanism of pork quality formation, especially non-coding RNAs (ncRNA) $[2,14,15]$. For example, miR-23a was identified to associate with the composite of slow myosin heavy chain isoforms [16], and miR-17-5p was found to reduce preadipocyte differentiation by inhibiting some targets such as FABP4 and PPARG [17]. In addition, IncRNA Inc_000414 was identified to closely associate with fat synthesis in Chinese Bamei pigs [18], and IncRNA MEG3 was identified to participate in the regulation of skeletal muscle development [19]. In circRNA, some circRNAs including 902, 937, 787 and 850 were identified to differentially express among differentiation stages of subcutaneous preadipocytes in Chinese Erhualian pigs [20]. The findings demonstrated that ncRNAs play roles in many aspects of pork quality formation. Despite advances in regulatory mechanism of pork quality formation, the molecular regulatory mechanism in pig muscle development remains unclear and little information is available.

The competing endogenous RNA (ceRNA) hypothesis was proposed as novel regulatory mechanism between coding messenger RNA (mRNA) and ncRNAs including miRNA, IncRNA and circRNA [21]. Many studies have demonstrated that IncRNA and circRNA as ceRNAs directly combine miRNA by miRNAresponse-elements (MREs) to indirectly regulate mRNA in various biological processes [22, 23]. Especially, ceRNA regulatory mechanisms are paid more attention on disease biology such as the occurrence and recurrence of myocardial infarction and tumor formation and metastasis [23-25]. Recently, a few ceRNArelated studies were investigated the roles in some economic traits in some economic animals. For example, Ma et al. found that IncRNA_Six1 as ceRNA participated in chicken myogenesis by regulating Six1 [26]. Wang et al. found that Inc_000679-miR221-5p-WNT3, Inc_000181-miR34a-GATA3 and Inc_000344-miR214-3p-SMAD3 were identified to play roles in hair follicle biology of Cashmere goat [27]. It is worth mentioning that a recent study has investigated the differences in muscle traits between Chinese indigenous Huainan pigs and Western commercial DLY crossbreed pigs, and identified some differentially expressed RNAs and regulatory factors such as miR423-5p and miR-874 [2]. Despite this, little ceRNA knowledge is available in pork quality formation, and more studies are required.

In this study, transcriptomes of 6 longissimus dorsi muscles from three SSs and three YYs with 90 100 $\mathrm{kg}$ live weight were sequenced using next-generation sequencing technology based on Illumina sequencing platform. Differentially expressed RNAs including IncRNA, circRNA, miRNA and mRNA were screened, and two ceRNA networks including IncRNA-miRNA-mRNA and circRNA-miRNA-mRNA were constructed, and quantitative real-time PCR (qRT-PCR) was performed to validate the sequencing results 
of gene expression. The study will provide novel insights into the regulatory mechanism of pork muscle formation between fat-type SS breed and lean-type YY breed.

\section{Methods}

\section{Animals and phenotypic determination}

Six barrows with 90 100 kg of live weight including three SSs and three YYs were used in this study. The SSs were purchased from the Saba pig breeding farm at Chuxiong city of the Yunnan Province, and the YYs were obtained from the pig breeding farm of the Yunnan Huijia Co. Ltd. All experimental pigs and procedures were approved by the Institutional Animal Care and Use Committee of Yunnan Agricultural University. Longissimus dorsi muscles were obtained from six pigs within fifteen minutes after slaughtered, and immediately frozen in liquid nitrogen to store for total RNA isolation. Meanwhile, longissimus dorsi tissues of $1 \times 1 \times 1 \mathrm{~cm}^{3}$ were processed using environment-friendly GD fixative (Servicebio, Wuhan, China) to measure muscle fiber diameter (MFD) by paraffin section. Other meat quality traits including meat color, marbling score, drip loss, water loss rate, cooked rate and intramuscular fat content (IMF) were determined according to the 'Technical regulation for determination of pork quality (NY/T 821-2004) of Agricultural Industry Standard of China.

\section{RNA preparation and sequencing}

The total RNA was extracted from longissimus dorsi tissues using TRIzol reagent (Invitrogen, CA, USA) according to the manufacturer's recommendations, and the quality of RNA was detected using $1.0 \%$ agarose gel electrophoresis and Qubit RNA Assay Kit in Qubit 2.0 Flurometer (Life, CA, USA), respectively. The RNA integrity number (RIN) was determined using the Agilent Bioanalyzer 2100 system (CA, USA). A total of $3 \mu \mathrm{g}$ RNA per sample was used to construct small RNA sequencing library using the NEBNext Multiplex Small RNA Library Prep Set (NEB, MA, USA), and index codes were added to attribute sequences to each sample. After the clustering of the index-coded samples were produced on a cBot Cluster Generation System using TruSeq SR Cluster Kit v3-cBot-HS (Illumia, CA, USA), and the RNA library was sequenced using Illumina Hiseq 2500 platform with 50 bp single-end (SE50) sequencing method. Similarly, Ribo-Zero RNA sequencing library was generated by $3 \mu \mathrm{g}$ RNAs per sample. Firstly, ribosomal RNA was removed by Epicentre Ribo-Zero rRNA Removal Kit (Epicentre, WI, USA). Subsequently, the strand-specific library was generated using the rRNA-depleted RNA by NEBNext Ultra Directional RNA Library Prep Kit for Illumina (NEB, MA, USA) according to manufacturer's instructions. Then, the clustering of the index-coded sample was performed on a cBot Cluster Generation System using TruSeq PE Cluster Kit v3-cBot-HS (Illumia, CA, USA). The library was sequenced on an Illumina Hiseq X Ten platform and 150 bp paired-end (PE150) reads were generated.

\section{Data analysis}

All raw data were filtered to generate clean data according to quality scores of all reads, and clean data were used to perform bioinformatics analysis including mapping, assembly, differentially expressed gene 
analysis, target gene prediction and functional enrichment analysis.

For miRNA-seq clean data, length-filtered reads were aligned to the reference genome (Sscrofa11.1, $\mathrm{ftp}$ ://ftp.ensembl.org/pub/release-92/fasta/sus_scrofa/dna/) using Bowtie2.0 software [28]. Known miRNAs were identified using mirdeep2.0 software on the basis of miRBase database (version 22.0, http://www.mirbase.org/), and novel miRNAs were predicted according to the secondary hairpin structure using miREvo1.1 and mirdeep2.0 software [29]. The miRNA expressions were analyzed using TPM (transcripts per million reads), and differentially expressed miRNAs (DEmiRNAs) between longissimus dorsi tissues from two pig breeds were analyzed using DESeq2.0 package in Bioconductor project [30], and $q$ value $<0.05$ was set as cut off criterion. DEmiRNA target genes were predicted using miRanda (http://www.microrna.org/), PITA and RNAhybrid software [31].

For Ribo-Zero RNA sequencing data, filtered paired-end reads were mapped across the pig genome (Sscrofa11.1) using Bowtie2.0 and HISAT2.0 packages with default parameters [28, 32], and the mapped reads were assembled using StringTie1.3.1 software [32]. The novel IncRNAs were identified according to the following: (1) transcripts with length shorter than $200 \mathrm{nt}$ and less than 2 exons were excluded; (2) transcripts were discarded by overlapping with annotated exon region; (3) transcripts with FPKM (fragments per kilobase of transcript sequence per millions base pairs sequenced) $<0.5$ were abandoned; (4) transcripts predicted with coding potential were filtered using the $\mathrm{CNCl}, \mathrm{CPC}$, Pfam-Scan and PhyloCSF tools [33-35]. Putative circRNAs were predicted using find_circ1.1 and CIRI2.1.2 packages with default parameters [36,37], and the expressions of circRNAs were quantified using TPM. Differentially expressed IncRNAs (DEIncRNAs) and circRNAs (DEcircRNAs) in longissimus dorsi tissues between SSs and YYs were screened using Ballgown and DESeq2.0 packages [30, 32], and $q$ value $<0.05$ were set as statistical significant thresholds. The targets of DEIncRNA were identified based on trans (co-expression) role, and the DEcircRNA host genes were obtained according to one-to-one match between circRNA and its host genes. The functional enrichment analyses of these DEmRNAs were performed using GOseq2.12 and KOBAS2.0 software with $q$ value $<0.05$ or $p$ value $<0.05$ cut off criterion $[38,39]$.

\section{Competitive endogenous RNA network construction}

The IncRNA-miRNA-mRNA and circRNA-miRNA-mRNA ceRNA networks were established on the basis of ceRNA hypothesis that IncRNA and circRNA directly combine miRNA by acting as miRNA sponge to indirectly regulate the function of mRNA [21]. The IncRNA-miRNA-mRNA and circRNA-miRNA-mRNA ceRNA networks were constructed and visualized using an open-source Cytoscape (version 3.7.0, https://cytoscape.org/) software [40].

\section{Quantitative real-time PCR validation}

To validate accuracy of RNA expression, quantitative real-time PCR (qRT-PCR) was performed for six samples including three SSs and three YYs. The CDNA was firstly reversely transcribed from total RNA using RevertAid First Strand cDNA Synthesis Kit (Thermo Fisher, MA, USA), and qRT-PCR was implemented using ABI Step one plus platform with FastStart Universal SYBR Green Master (Roche, 
Basel, Switzerland) according to the manufacturer's recommendations. Each sample was analyzed in triplicate, and the $2^{-\Delta \Delta C t}$ method was used evaluate to the RNA relative expression.

\section{Results}

\section{Pork quality performance}

To investigate the difference in muscle between fat-type SS and lean-type YY, pork quality traits in longissimus dorsi muscle were detected. The results showed that there was no significant difference in average live weight between SSs $(94.17 \pm 5.51 \mathrm{~kg})$ and YYs $(96.67 \pm 3.06 \mathrm{~kg})(P>0.05)$ at slaughter. Compared to YYs, SSs had higher marbling score and intramuscular fat content $(P<0.05)$, and lower drip loss, water loss rate, and muscle fiber diameter $(P<0.05$, Fig. 1, Table 1$)$, which indicated the differences in pork quality between two pig breeds.

\section{Landscape of RNA sequencing data}

Briefly, 10222987 12553881 raw single-end reads were obtained in the small RNA libraries of 6 longissimus dorsi muscle tissues from SSs and YYs. The base error rate was below $0.01 \%$ and data with $>$ Q30 was above $93 \%$. Clean reads filtered was over $97 \%$, and more than $95 \%$ length-filtered reads were mapped to reference genome (Sscrofa11.1, Table 2). For Ribo-Zero RNA libraries, a total of 84433622 103454610 clean paired-end reads with Q30 $>92 \%$ and valid ratio $>97 \%$ were produced by six longissimus dorsi muscle tissues between two pig breeds, and over $91 \%$ of valid reads were aligned to pig reference genome (Table 3 ).

\section{Differential expression analysis}

All raw data were subjected to quality control, and a total of 383 miRNAs ( 343 known miRNAs and 40 novel miRNAs), 45788 mRNAs, 11591 IncRNAs (276 annotated IncRNAs and 11315 novel IncRNAs) and 5124 circRNAs were obtained (Table S1 4). According to $q$ value $<0.05$ or $p$ value $<0.05,31$ DEmiRNAs (11 up- and 20 down- regulated), 902 DEmRNAs (460 up- and 442 down- regulated), 47 DElncRNAs (28 up- and 19 down- regulated) and 198 DEcircRNAs (73 up- and 125 down- regulated) were identified in longissimus dorsi muscles between SS and YY breeds (Table S5 8). The distributions of DEmiRNAs, DEmRNAs, DElncRNAs and DEcircRNAs were separately displayed using volcano plots in Fig. 2A D. The expression levels of DEmiRNAs, DEmRNAs, DElncRNAs and DEcircRNAs in longissimus dorsi muscles between SS and YY breeds were displayed using heat map in Fig. 3A D.

\section{Functional enrichment analysis}

To better understand the functional roles of 902 DEmRNAs between SS and YY breeds, GO including biological process (BP), cellular component (CC) as well as molecular function (MF), and KEGG pathway analyses of 902 DEmRNAs were performed. The GO results showed that 2 BPs, 5 CCs and 3 MFs were significantly enriched (Fig. 4A). Two BPs were DNA-dependent transcription and initiation and oxidation- 
reduction process, separately. Five CCs were intracellular part, cytoplasm, intracellular, organelle and intracellular organelle, separately. Three MFs were separately cofactor binding, coenzyme binding and oxidoreductase activity.

KEGG pathway analysis showed that 20 KEGG pathways were significantly enriched (Table S9), and 20 KEGG pathways were showed in Fig. 4B. The 20 KEGG pathways were mainly associated with glycolysis/gluconeogenesis, amino acid biosynthesis, carbohydrate and organic acid metabolism.

\section{CeRNA network construction}

To elucidate the interactive relationships of DElncRNAs DEcircRNAs, DEmiRNAs and mRNAs, two ceRNA networks including IncRNA-miRNA-mRNA and circRNA-miRNA-mRNA were established on the basis of ceRNA hypothesis, and visualized by Cytoscape software (Fig. 5). In IncRNA-miRNA-mRNA ceRNA network, 21 IncRNAs (14 up- and 7 down- regulated), 10 miRNA (5 up- and 5 down- regulated) and 52 mRNAs (34 up- and 18 down- regulated) were contained, and miR-1343 (degree=16), CCDC107 (degree=16) and ALDBSSCG0000001163 (degree=16) had the most links in miRNAs, mRNAs and IncRNAs in whole ceRNA (Fig. 5A). In circRNA-miRNA-mRNA ceRNA network, 46 circRNAs (17 up- and 29 down- regulated), 8 miRNAs (4 up- and 4 down- regulated) and 36 mRNAs (25 up- and 11 downregulated) were contained (Fig. 5B). In ceRNA-circRNA network, miR-1343 (degree=28), novel_circ_0008532 (degree=3) and MAVS and SLC16A5 (degree=2) had the most links in miRNAs, circRNAs and mRNAs, separately (Fig. 5B). MiRNA miR-1343 was identified to have the highest degree in both ceRNA networks, which indicated that miR-1343 might play key regulatory roles in pork quality formation.

\section{Gene expression validation by qRT-PCR}

To confirm the accuracy of identified DERNAs, qRT-PCR was performed for 8 randomly selected DEGs to validate the differentially expression analysis results, including 4 down-regulated miRNAs (miR-10383, 362, 874 and 432), 2 up-regulated circRNAs (circ-0003500 and 0001264), 1 up-regulated IncRNA (Inc011218) and 1 up-regulated mRNA (SLC16A5) in ceRNA network. The qRT-PCR primers are presented in

Table S10. The results showed that sequencing data and qRT-PCR exhibited the consistent tendencies in expression (Fig. 6), which indicated that the sequencing data was reliable in our study.

\section{Discussion}

Pork quality is an important economic trait in pig industry, and many studies have demonstrated that the pork formation has significant differences between Chinese indigenous pig breeds and Western mainly commercial pig breeds $[3,13,41]$. Despite some advances in molecular genetic mechanism $[3,9,12,13$, 42], the regulatory mechanism of pork quality formation remains unclear. Uncovering the regulatory mechanism involved in pork formation contributes to better utilizing Chinese and Western pig resources and producing crossbred pig breed with better performances such as higher pork quality and faster growth. NcRNAs are non-coding parts of genome at different cell status, and account for more than $90 \%$ 
RNA transcripts [43]. Increasing data have demonstrated that ncRNAs played the important regulatory roles in many biological processes such as sex differentiation [44], organ development [45], and tumor origination and progression [46]. More and more studies revealed that ncRNAs played regulatory roles by ceRNA network on the basis of ceRNA hypothesis [21], and utilizing ceRNA network analysis have identified a lot of regulatory factors including IncRNAs, miRNAs and cirRNAs associated with some animal economic traits such as hair follicle cycle of cashmere goat and poultry meat quality $[27,47]$. Although some studies also have paid attention on ceRNA regulatory mechanism in muscle of pig $[2,48]$, little information is available in ceRNA regulatory pork quality formation. In this study, fat-type SS with higher pork quality and lean-type YY with faster growth as two typical pig breeds were used to investigate the regulatory mechanism of pork quality formation by transcriptome analysis including screening differentially expressed RNA and constructing ceRNA network. Finally, 31 DEmiRNAs, 902 DEmRNAs, 47 DElncRNAs and 198 DEcircRNAs were identified in longissimus dorsi muscles between SSs and YYs. Two ceRNA networks including IncRNA-miRNA-mRNA and circRNA-miRNA-mRNA revealed that miR-1343 was identified to play important regulatory role in longissimus dorsi muscle formation between two pig breeds.

MicroRNA miR-1343 has so far been identified to play roles in regulating the fibrosis of human and the pluripotency of porcine pluripotent stem cells $[49,50]$, and some studies showed that miR-1343 was related with the susceptible to porcine reproductive and respiratory syndrome (PRRS) and pig backfat formation [51, 52]. So far, no studies clearly reported the function of miR-1343 in pork quality formation. This study was firstly to identify miR-1343 played importantly regulatory role in pork quality formation between fat-type and lean-type pig breeds by two ceRNA networks. In IncRNA-related and circRNA-related ceRNA networks, down-regulated IncRNA and circRNAs combined up-regulated miR-1343 were XLOC_375095, and circ_0020383, 0020132, 0003861, 0002904 and 0004019, separately. Common mRNA down-regulated by miR-1343 was $E N H O$. ENHO is an energy homeostasis associated gene that encodes a peptide hormone adropin with differentially expression in various tissues [53]. The function of adropin is mainly to maintain energy homeostasis and insulin response [53], and has been identified to associated with many diseases such as systemic sclerosis [54], rheumatoid arthritis and systemic lupus ergthematosus [55]. Recent studies showed that adropin was identified to participate the regulation of muscle substrate preference and pork production of pig $[53,56]$, which indicated that $E N H O$ was associated with pork quality formation. This study demonstrated that down-regulated IncRNA and circRNAs contributed to significantly up-regulating miR-1343 to down-regulate $E N H O$, which play regulatory roles in longissimus dorsi muscle formation of SSs and YYs.

Although miR-1343 has been identified to play important regulatory roles in pork quality formation and is potentially valuable in pig breeding industry, the limitation must be noted. The microRNA miR-1343 was identified by transcriptome sequencing and bioinformatics analysis. More functional studies are required to investigate the roles of miR-1343 in pork quality formation by experiments.

\section{Conclusions}


The present study identified miR-1343 as key regulatory factor in pig longissimus dorsi muscle formation by ceRNA networks, which provides novel insights into molecular regulatory mechanism of pork quality formation between fat-type and lean-type pig breeds.

\section{Abbreviations}

SS: Saba pig; YY: Yorkshire pig; ncRNAs: non-coding RNAs; ceRNA: Competing endogenous RNA; MRE: miRNA response element; MFD: Muscle fiber diameter; IMF: Intramuscular fat content; RIN: RNA integrity number; TPM: Transcript per million; DEmiRNAs: Differentially expressed miRNAs; FPKM: Fragments per kilobase of transcript sequence per millions base pairs sequenced; DElncRNA: Differentially expressed IncRNA; DEcircRNA: Differentially expressed circRNA; DEmRNA: Differentially expressed mRNA; GO: Gene Ontology; BP: biological process; CC: cellular component; MF: molecular function; KEGG: Kyoto Encyclopedia of Genes and Genomes; CCDC107: coiled-coil domain containing 107; MAVS. Mitochondrial antiviral signaling protein; SLC16A5: Solute carrier family 16 member 5; ENHO: energy homeostasis associated

\section{Declarations}

\section{Ethics approval and consent to participate}

All animal experiments procedures were approved by the Institutional Animal Care and Use Committee (IACUC) of Yunnan Agricultural University. All of the experiments were performed in accordance with the relevant guidelines and regulations by the Ministry of Agriculture and Rural Affairs of China.

\section{Consent for publication}

Not Applicable.

\section{Availability of data and materials}

All data analyzed in this study are included in the article and its Additional files; and the raw reads were deposited in Sequence Read Archive (https://submit.ncbi.nlm.nih.gov/subs/sra/) with the accession number PRJNA514228.

\section{Competing interests}

The authors declare that they have no conflict of interests.

\section{Funding}

This work was financially supported by National Key R\&D Program (no 2018YFD0501206), Yunnan Swine Industry Technology System Program (2019KJTX0013), and National Natural Science 
Foundations of China (no U1402266). The funders of this study had no role in study design, data collection and analysis, decision to publish, or preparation of the manuscript.

\section{Authors' contributions}

SXL conceived and designed this study, and revised the manuscript. MLL, DWY, XXD, GXL and YY provided powerful assistance for the experiment including phenotype determination, sample collection and manuscript revision. WH and QC wrote the manuscript. WH, XYW and QC analyzed the data. All authors have read and approved the final manuscript.

\section{Acknowledgements}

We are grateful to the members of this laboratory for their constructive comments and strong revisions of this manuscript, especially Jingru Nie. We thank our collaborators, Mr. Yanlin Zhang and Mr. Wenhui Ren for their assistance in sample collection.

\section{Author details}

${ }^{1}$ Faculty of Animal Science and Technology, Yunnan Agricultural University, Kunming 650201, China.

${ }^{2}$ Key Laboratory of Animal Nutrition and Feed Science of Yunnan Province, Yunnan Agricultural University, Kunming 650201, China.

\section{References}

1. Nonneman DJ, Shackelford SD, King DA, Wheeler TL, Wiedmann RT, Snelling WM, et al. Genome-wide association of meat quality traits and tenderness in swine. J Anim Sci. 2013;91(9):4043-50.

2. Wang J, Ren Q, Hua L, Chen J, Zhang J, Bai H, et al. Comprehensive Analysis of Differentially Expressed mRNA, IncRNA and circRNA and Their ceRNA Networks in the Longissimus Dorsi Muscle of Two Different Pig Breeds. Int J Mol Sci. 2019;20(5):1107.

3. Liu X, Xiong X, Yang J, Zhou L, Yang B, Ai H, et al. Genome-wide association analyses for meat quality traits in Chinese Erhualian pigs and a Western Duroc $\mathrm{x}$ (Landrace $\mathrm{x}$ Yorkshire) commercial population. Genet Sel Evol. 2015;47:44.

4. Rosenvold K, Andersen HJ. Factors of significance for pork quality-a review. Meat Sci. 2003;64(3):219-37.

5. Li HD, Lund MS, Christensen OF, Gregersen VR, Henckel P, Bendixen C. Quantitative trait loci analysis of swine meat quality traits. J Anim Sci. 2010;88(9):2904-12.

6. Andersson L, Georges M. Domestic-animal genomics: deciphering the genetics of complex traits. Nat Rev Genet. 2004;5(3):202-12.

7. Fujii J, Otsu K, Zorzato F, de Leon S, Khanna VK, Weiler JE, et al. Identification of a mutation in porcine ryanodine receptor associated with malignant hyperthermia. Science. 1991;253(5018):44851. 
8. Uimari P, Sironen A. A combination of two variants in PRKAG3 is needed for a positive effect on meat quality in pigs. BMC Genet. 2014;15:29.

9. Zhang C, Wang Z, Bruce H, Kemp RA, Charagu P, Miar Y, et al. Genome-wide association studies (GWAS) identify a QTL close to PRKAG3 affecting meat $\mathrm{pH}$ and colour in crossbred commercial pigs. BMC Genet. 2015;16:33.

10. Carrodeguas JA, Burgos C, Moreno C, Sanchez AC, Ventanas S, Tarrafeta L, et al. Incidence in diverse pig populations of an IGF2 mutation with potential influence on meat quality and quantity: An assay based on real time PCR (RT-PCR). Meat Sci. 2005;71(3):577-82.

11. Herault F, Damon M, Cherel P, Le Roy P. Combined GWAS and LDLA approaches to improve genomewide quantitative trait loci detection affecting carcass and meat quality traits in pig. Meat Sci. 2018;135:148-58.

12. Zhang Y, Zhang J, Gong H, Cui L, Zhang W, Ma J, et al. Genetic correlation of fatty acid composition with growth, carcass, fat deposition and meat quality traits based on GWAS data in six pig populations. Meat Sci. 2019;150:47-55.

13. Xiong X, Liu X, Zhou L, Yang J, Yang B, Ma H, et al. Genome-wide association analysis reveals genetic loci and candidate genes for meat quality traits in Chinese Laiwu pigs. Mamm Genome. 2015;26(3-4):181-90.

14. Kumar H, Srikanth K, Park W, Lee SH, Choi BH, Kim H, et al. Transcriptome analysis to identify long non coding RNA (IncRNA) and characterize their functional role in back fat tissue of pig. Gene. 2019;703:71-82.

15. Wei W, Li B, Liu K, Jiang A, Dong C, Jia C, et al. Identification of key microRNAs affecting drip loss in porcine longissimus dorsi by RNA-Seq. Gene. 2018;647:276-82.

16. Shen L, Chen L, Zhang S, Zhang Y, Wang J, Zhu L. MicroRNA-23a reduces slow myosin heavy chain isoforms composition through myocyte enhancer factor 2C (MEF2C) and potentially influences meat quality. Meat Sci. 2016;116:201-6.

17. Han H, Gu S, Chu W, Sun W, Wei W, Dang X, et al. miR-17-5p Regulates Differential Expression of NCOA3 in Pig Intramuscular and Subcutaneous Adipose Tissue. Lipids. 2017;52(11):939-49.

18. Sun Y, Chen X, Qin J, Liu S, Zhao R, Yu T, et al. Comparative Analysis of Long Noncoding RNAs Expressed during Intramuscular Adipocytes Adipogenesis in Fat-Type and Lean-Type Pigs. J Agric Food Chem. 2018;66(45):12122-30.

19. Yu X, Wang Z, Sun H, Yang Y, Li K, Tang Z. Long non-coding MEG3 is a marker for skeletal muscle development and meat production traits in pigs. Anim Genet. 2018;49(6):571-8.

20. Liu X, Liu K, Shan B, Wei S, Li D, Han H, et al. A genome-wide landscape of mRNAs, IncRNAs, and circRNAs during subcutaneous adipogenesis in pigs. J Anim Sci Biotechnol. 2018;9:76.

21. Salmena L, Poliseno L, Tay Y, Kats L, Pandolfi PP. A ceRNA hypothesis: the Rosetta Stone of a hidden RNA language? Cell. 2011;146(3):353-8.

22. Wei Y, Chang Z, Wu C, Zhu Y, Li K, Xu Y. Identification of potential cancer-related pseudogenes in lung adenocarcinoma based on ceRNA hypothesis. Oncotarget. 2017;8(35):59036-47. 
23. Zhang G, Sun H, Zhang Y, Zhao H, Fan W, Li J, et al. Characterization of dysregulated IncRNA-mRNA network based on ceRNA hypothesis to reveal the occurrence and recurrence of myocardial infarction. Cell Death Discov. 2018;4:35.

24. Li $\mathrm{Y}$, Wang $\mathrm{H}$, Huang $\mathrm{H}$. Long non-coding RNA MIR205HG function as a ceRNA to accelerate tumor growth and progression via sponging miR-122-5p in cervical cancer. Biochem Biophys Res Commun. 2019;514(1):78-85.

25. Li Z, Jiang P, Li J, Peng M, Zhao X, Zhang X, et al. Tumor-derived exosomal Inc-Sox2ot promotes EMT and stemness by acting as a ceRNA in pancreatic ductal adenocarcinoma. Oncogene. 2018;37(28):3822-38.

26. Ma M, Cai B, Jiang L, Abdalla BA, Li Z, Nie Q, et al. IncRNA-Six1 Is a Target of miR-1611 that Functions as a ceRNA to Regulate Six1 Protein Expression and Fiber Type Switching in Chicken Myogenesis. Cells. 2018;7:12.

27. Wang S, Ge W, Luo Z, Guo Y, Jiao B, Qu L, et al. Integrated analysis of coding genes and non-coding RNAs during hair follicle cycle of cashmere goat (Capra hircus). BMC Genomics. 2017;18:767.

28. Langdon WB. Performance of genetic programming optimised Bowtie2 on genome comparison and analytic testing (GCAT) benchmarks. BioData Min. 2015;8:1.

29. Wen M, Shen Y, Shi S, Tang T. miREvo: an integrative microRNA evolutionary analysis platform for next-generation sequencing experiments. BMC Bioinformatics. 2012;13:140.

30. Love MI, Huber W, Anders S. Moderated estimation of fold change and dispersion for RNA-seq data with DESeq2. Genome Biol. 2014;15(12):550.

31. Rehmsmeier M, Steffen P, Hochsmann M, Giegerich R. Fast and effective prediction of microRNA/target duplexes. RNA. 2004;10(10):1507-17.

32. Pertea M, Kim D, Pertea GM, Leek JT, Salzberg SL. Transcript-level expression analysis of RNA-seq experiments with HISAT, StringTie and Ballgown. Nat Protoc. 2016;11(9):1650-67.

33. Lin MF, Jungreis I, Kellis M. PhyloCSF: a comparative genomics method to distinguish protein coding and non-coding regions. Bioinformatics. 2011;27(13):i275-82.

34. Bateman A, Coin L, Durbin R, Finn RD, Hollich V, Griffiths-Jones S, et al. The Pfam protein families database. Nucleic Acids Res. 2004;32:D138-41.

35. Mukherjee S, Manna S, Mukherjee P, Panda CK. Differential alterations in metabolic pattern of the spliceosomal uridylic acid-rich small nuclear RNAs (UsnRNAs) during malignant transformation of 20-methylcholanthrene-induced mouse CNCI-PM-20 embryonic fibroblasts. Mol Carcinog. 2009;48(9):773-8.

36. Memczak S, Jens M, Elefsinioti A, Torti F, Krueger J, Rybak A, et al. Circular RNAs are a large class of animal RNAs with regulatory potency. Nature. 2013;495(7441):333-8.

37. Gao Y, Wang J, Zhao F. CIRI: an efficient and unbiased algorithm for de novo circular RNA identification. Genome Biol. 2015;16:4. 
38. Xie C, Mao X, Huang J, Ding Y, Wu J, Dong S, et al. KOBAS 2.0: a web server for annotation and identification of enriched pathways and diseases. Nucleic Acids Res. 2011;39:W316-22.

39. Young MD, Wakefield MJ, Smyth GK, Oshlack A. Gene ontology analysis for RNA-seq: accounting for selection bias. Genome Biol. 2010;11(2):R14.

40. Shannon P, Markiel A, Ozier O, Baliga NS, Wang JT, Ramage D, et al. Cytoscape: a software environment for integrated models of biomolecular interaction networks. Genome Res. 2003;13(11):2498-504.

41. Ma J, Yang J, Zhou L, Zhang Z, Ma H, Xie X, et al. Genome-wide association study of meat quality traits in a White DurocxErhualian F2 intercross and Chinese Sutai pigs. PLoS One. 2013;8(5):e64047.

42. Casiro S, Velez-Irizarry D, Ernst CW, Raney NE, Bates RO, Charles MG, et al. Genome-wide association study in an F2 Duroc $x$ Pietrain resource population for economically important meat quality and carcass traits. J Anim Sci. 2017;95(2):545-58.

43. Consortium EP. An integrated encyclopedia of DNA elements in the human genome. Nature. 2012;489(7414):57-74.

44. Tao W, Sun L, Shi H, Cheng Y, Jiang D, Fu B, et al. Integrated analysis of miRNA and mRNA expression profiles in tilapia gonads at an early stage of sex differentiation. BMC Genomics. 2016;17:328.

45. Wei Q, Lei R, Hu G. Roles of miR-182 in sensory organ development and cancer. Thorac Cancer. 2015;6(1):2-9.

46. Fitzpatrick C, Bendek MF, Briones M, Farfan N, Silva VA, Nardocci G, et al. Mitochondrial ncRNA targeting induces cell cycle arrest and tumor growth inhibition of MDA-MB-231 breast cancer cells through reduction of key cell cycle progression factors. Cell Death Dis. 2019;10(6):423.

47. Zhang M, Li F, Sun JW, Li DH, Li WT, Jiang RR, et al. LncRNA IMFNCR Promotes Intramuscular Adipocyte Differentiation by Sponging miR-128-3p and miR-27b-3p. Front Genet. 2019;10:42.

48. Sun J, Xie M, Huang Z, Li H, Chen T, Sun R, et al. Integrated analysis of non-coding RNA and mRNA expression profiles of 2 pig breeds differing in muscle traits. J Anim Sci. 2017;95(3):1092-103.

49. Stolzenburg LR, Wachtel S, Dang H, Harris A. miR-1343 attenuates pathways of fibrosis by targeting the TGF-beta receptors. Biochem J. 2016;473(3):245-56.

50. Xie Y, Cao H, Zhang Z, Zhang S, Wang H. Molecular network of miR-1343 regulates the pluripotency of porcine pluripotent stem cells via repressing OTX2 expression. RNA Biol. 2019;16(1):82-92.

51. Zhen Y, Wang F, Liang W, Liu J, Gao G, Wang Y, et al. Identification of Differentially Expressed Noncoding RNA in Porcine Alveolar Macrophages from Tongcheng and Large White Pigs Responded to PRRSV. Sci Rep. 2018;8:15621.

52. Chen C, Deng B, Qiao M, Zheng R, Chai J, Ding Y, et al. Solexa sequencing identification of conserved and novel microRNAs in backfat of Large White and Chinese Meishan pigs. PLoS One. $2012 ; 7(2): e 31426$. 
53. Wang SP, Gao YL, Liu G, Deng D, Chen RJ, Zhang YZ, et al. Molecular cloning, characterization and expression of the energy homeostasis-associated gene in piglet. J Zhejiang Univ Sci B. 2015;16(6):524-32.

54. Yolbas S, Kara M, Yilmaz M, Aydin S, Koca SS. Serum adropin level and ENHO gene expression in systemic sclerosis. Clin Rheumatol. 2016;35(6):1535-40.

55. Yolbas S, Kara M, Kalayci M, Yildirim A, Gundogdu B, Aydin S, et al. ENHO gene expression and serum adropin level in rheumatoid arthritis and systemic lupus erythematosus. Adv Clin Exp Med. 2018;27(12):1637-41.

56. Gao S, McMillan RP, Jacas J, Zhu Q, Li X, Kumar GK, et al. Regulation of substrate oxidation preferences in muscle by the peptide hormone adropin. Diabetes. 2014;63(10):3242-52.

\section{Tables}

Table 1 Comparison of pork quality traits between Saba and Yorkshire pigs (LSM \pm SE)

\begin{tabular}{|c|c|c|c|c|c|c|c|c|c|}
\hline \multicolumn{2}{|c|}{$\begin{array}{c}\text { BreedWeight } \\
\text { (kg) }\end{array}$} & $\mathrm{pH}_{1}$ & $\begin{array}{l}\text { Color } \\
\text { score } \\
\end{array}$ & $\begin{array}{l}\text { Marbling } \\
\text { score }\end{array}$ & $\begin{array}{l}\text { Water loss rate } \\
(\%)\end{array}$ & $\begin{array}{l}\text { Cooked rate } \\
\text { (\%) }\end{array}$ & $\begin{array}{l}\text { Drip loss } \\
(\%)\end{array}$ & IMF (\%) & MFD $(\mu \mathrm{m})$ \\
\hline$\overline{\mathrm{SS}}$ & $94.17 \pm 5$ & 516.42 & $23.83 \pm 0$ & $94.00 \pm 0.50$ & ${ }^{*} 13.45 \pm 1.60^{*}$ & $63.40 \pm 2.52$ & $1.47 \pm 0.51$ & ${ }^{*} 5.76 \pm 1.1$ & $1^{*} 47.38 \pm 2.60^{*}$ \\
\hline YY & $96.67 \pm 3$ & 66.48 & $33.33 \pm 0$ & $92.67 \pm 0.29$ & ${ }^{*} 20.59 \pm 3.79^{*}$ & $63.56 \pm 1.29$ & $2.19 \pm 0.51^{\prime}$ & * $3.21 \pm 0.5$ & $8^{*} 66.98 \pm 7.41^{*}$ \\
\hline
\end{tabular}

${ }^{*} P<0.05$. LSM, least squares mean; SE, standard error; IMF, intramuscular fat content; MFD, muscle fiber diameter.

Table 2 Overview of small RNA sequencing data in longissimus dorsi muscle between SS and YY pigs

\begin{tabular}{|c|c|c|c|c|c|c|c|c|}
\hline \multicolumn{2}{|c|}{ Sample Raw } & $\begin{array}{l}\text { Bases } \\
(\mathrm{Gb})\end{array}$ & \multirow{2}{*}{$\begin{array}{l}\begin{array}{l}\text { Error rate } \\
(\%)\end{array} \\
0.01\end{array}$} & \multirow{2}{*}{$\begin{array}{l}\begin{array}{l}\text { Q30 } \\
(\%)\end{array} \\
93.54\end{array}$} & \multirow{2}{*}{$\begin{array}{l}\text { Clean } \\
\text { reads } \\
10,336,729\end{array}$} & \multicolumn{3}{|c|}{ Valid ratio (\%) Mapped reads Mapped ratio (\%) } \\
\hline $\begin{array}{l}\text { SS_1 } \\
1\end{array}$ & 10,585 , & 0.53 & & & & 97.65 & $9,619,382$ & $\begin{array}{l}97.39 \\
\end{array}$ \\
\hline SS_2 & 12,553, & 0.69 & 0.01 & 93.18 & $12,307,771$ & 98.04 & $11,259,391$ & 95.45 \\
\hline SS_3 & 10,845, & 0.54 & 0.01 & 93.65 & $10,639,892$ & 98.10 & $10,107,860$ & 96.51 \\
\hline YY_1 & 11,828, & 30.59 & 0.01 & 93.64 & $11,598,297$ & 98.05 & $10,795,726$ & 95.77 \\
\hline YY_2 & 10,222, & 70.51 & 0.01 & 95.51 & $10,004,662$ & 97.86 & $9,350,059$ & 96.09 \\
\hline YY_3 & 10,936, & 10.55 & 0.01 & 95.73 & $10,792,306$ & 98.68 & $10,316,149$ & 97.05 \\
\hline
\end{tabular}

SS, Saba pig; YY, Yorkshire pig.

Table 3 Overview of Ribo-Zero RNA sequencing reads in longissimus dorsi muscle between SS and YY pigs 


\begin{tabular}{lllllllll}
\hline Sample Raw reads & $\begin{array}{l}\text { Error rate } \\
(\%)\end{array}$ & $\begin{array}{l}\text { Q30 } \\
(\%)\end{array}$ & $\begin{array}{l}\text { Clean reads Clean bases } \\
(\mathrm{Gb})\end{array}$ & $\begin{array}{l}\text { Valid ratio } \\
(\%)\end{array}$ & $\begin{array}{l}\text { Total } \\
\text { mapped }\end{array}$ & $\begin{array}{l}\text { Mapped ratio } \\
(\%)\end{array}$ \\
\hline SS_1 & $88,777,272$ & 0.01 & 92.87 & $86,751,052$ & 13.01 & 97.72 & $79,891,730$ & 92.09 \\
SS_2 & $86,286,930$ & 0.01 & 93.55 & $844,33,622$ & 12.67 & 97.85 & $76,997,549$ & 91.19 \\
\hline SS_3 & $91,976,404$ & 0.01 & 93.46 & $902,72,818$ & 13.54 & 98.15 & $83,490,713$ & 92.49 \\
\hline YY_1 & $105,818,2140.01$ & 93.52 & $103,454,61015.52$ & 97.77 & $95,166,411$ & 91.99 \\
YY_2 & $105,504,9120.01$ & 93.43 & $103,185,59815.48$ & 97.80 & $95,141,652$ & 92.20 \\
\hline YY_3 & $91,228,592$ & 0.01 & 93.01 & $88,554,636$ & 13.28 & 97.07 & $82,115,341$ & 92.73 \\
\hline
\end{tabular}

SS, Saba pig; YY, Yorkshire pig.

\section{Figures}


A

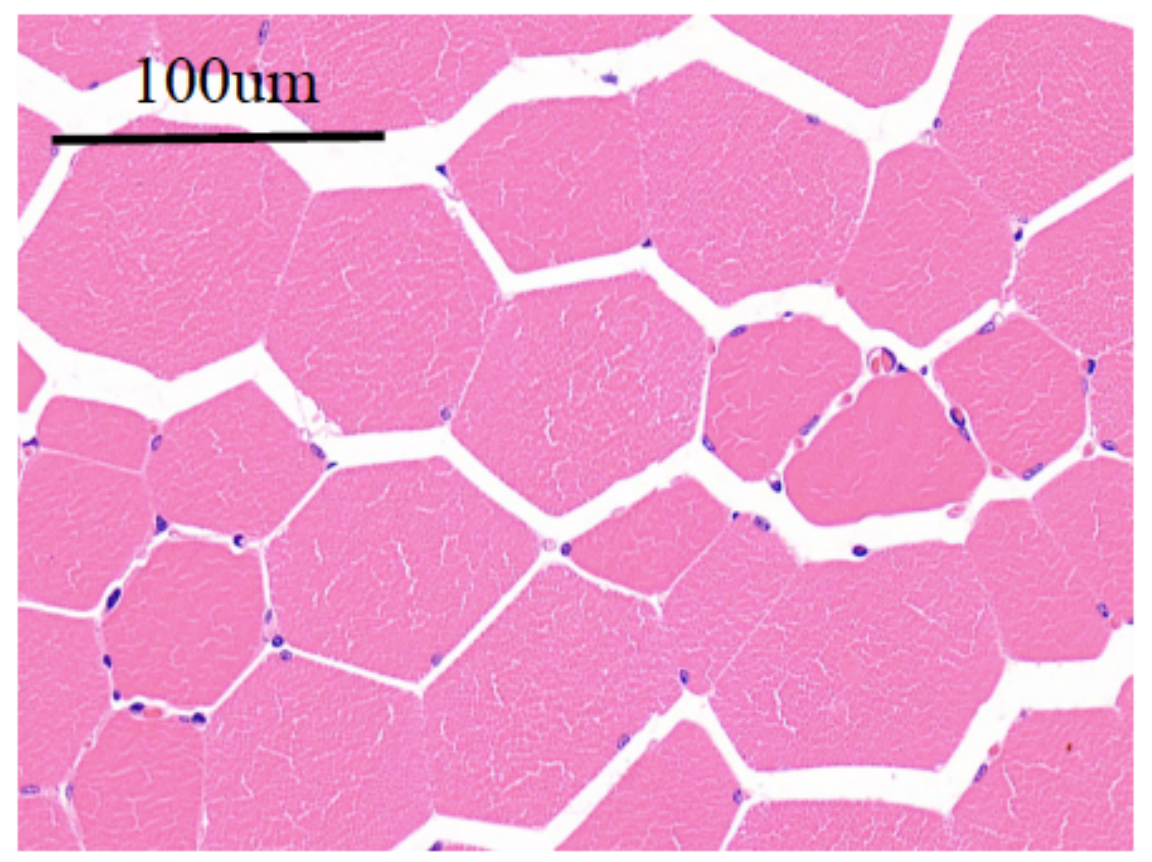

B

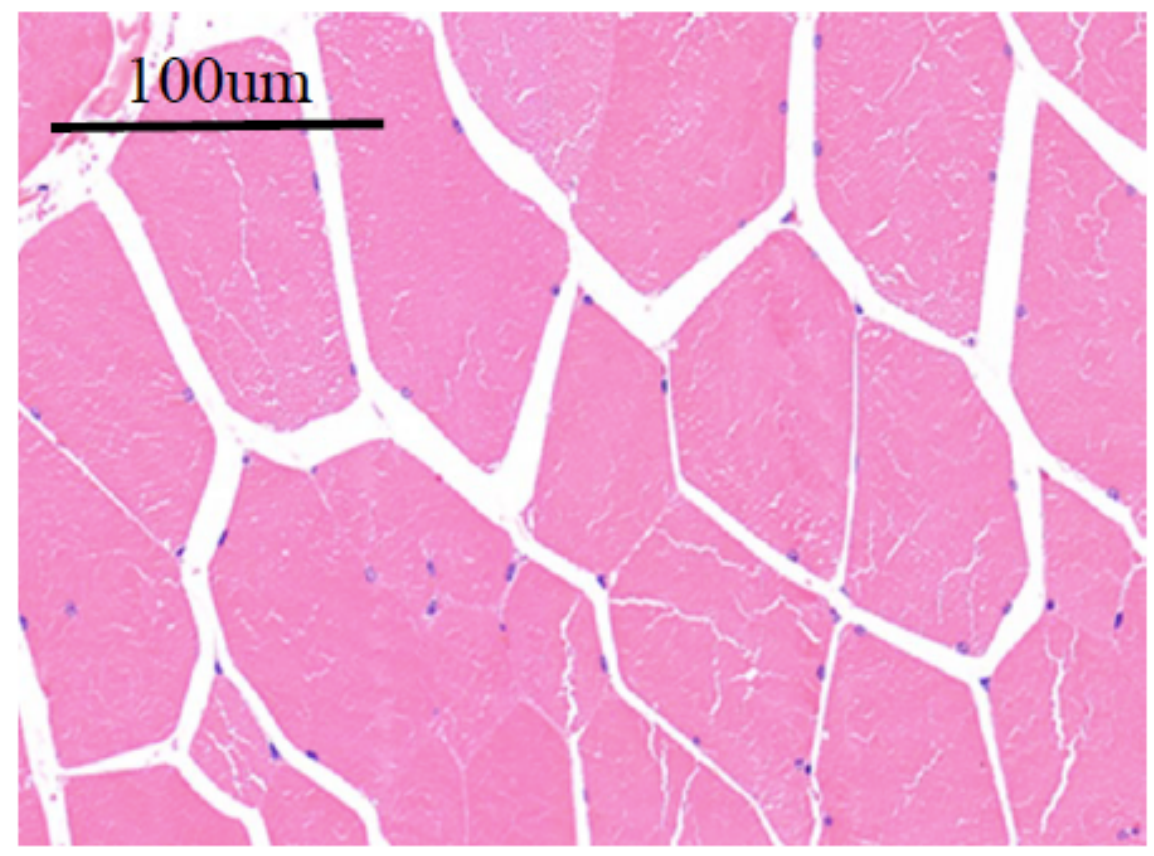

\section{Figure 1}

Paraffin section by hematoxylin-eosin staining of longissimus dorsi muscle between Saba and Yorkshire pigs. (A) Saba pig; (B) Yorkshire pig. Longissimus dorsi muscle of Saba pig had smaller muscle fiber diameter. 
A
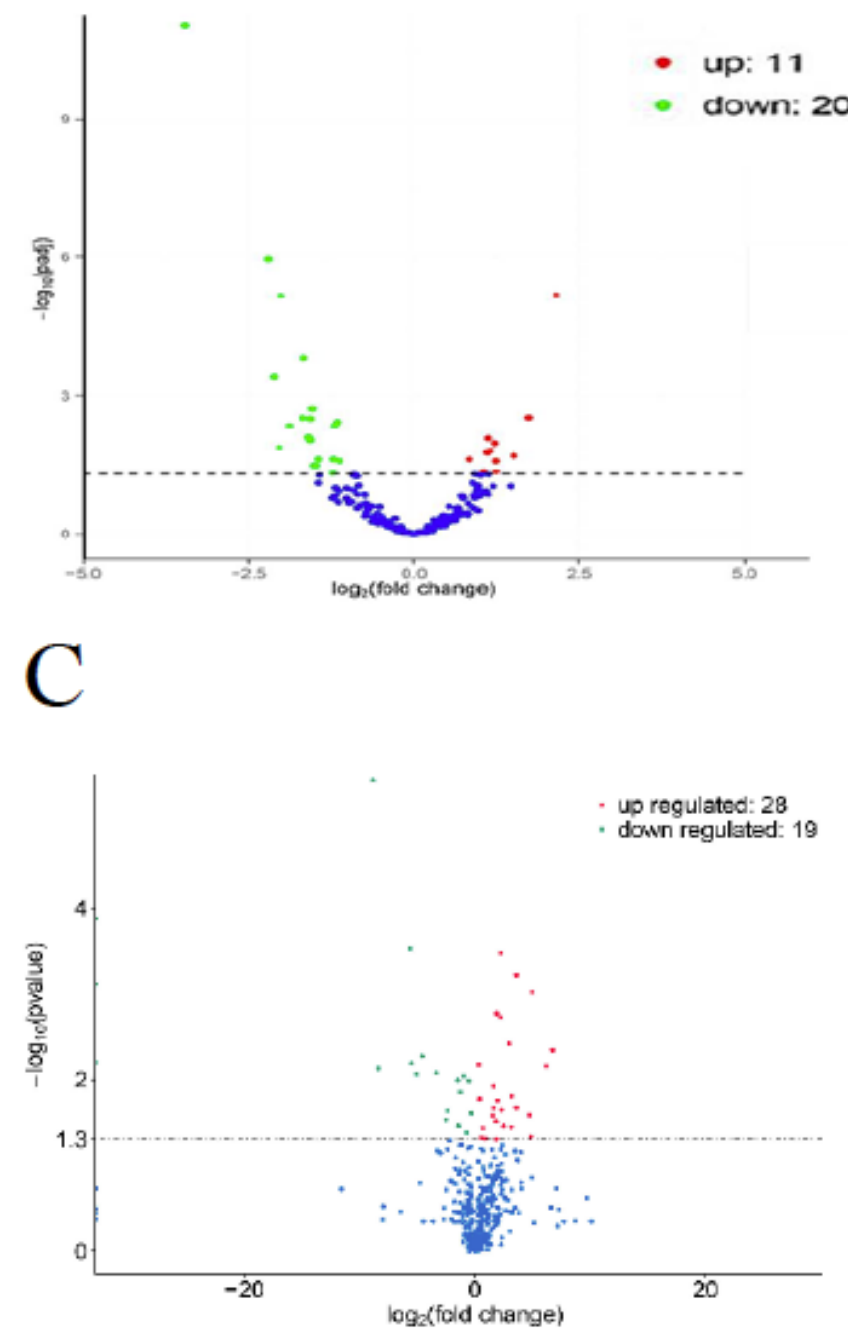

$\mathrm{B}$
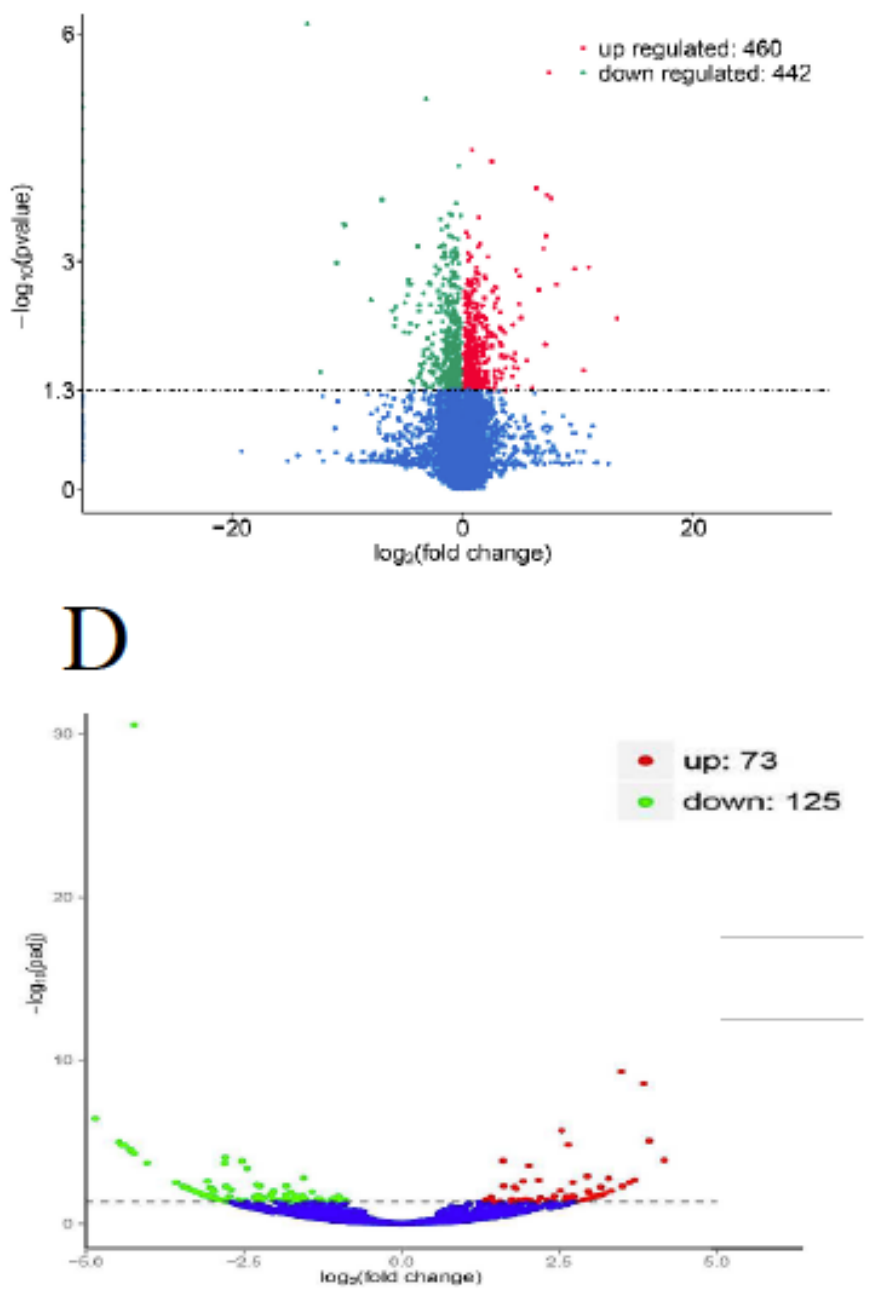

Figure 2

Volcano plot of expressions of four types of RNAs. (A) miRNA; (B) mRNA; (C) IncRNA and (D) circRNA. Horizontal axis represented the mean expression differences in longissimus dorsi muscle between Saba and Yorkshire pigs, and vertical axis represented log transformed FDR. FDR, false discovery rate. 


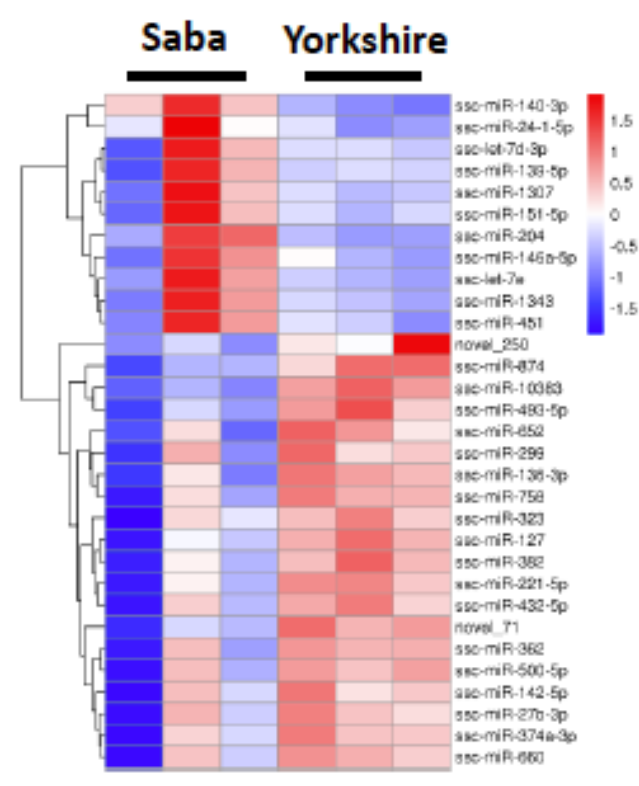

$\mathrm{C}$

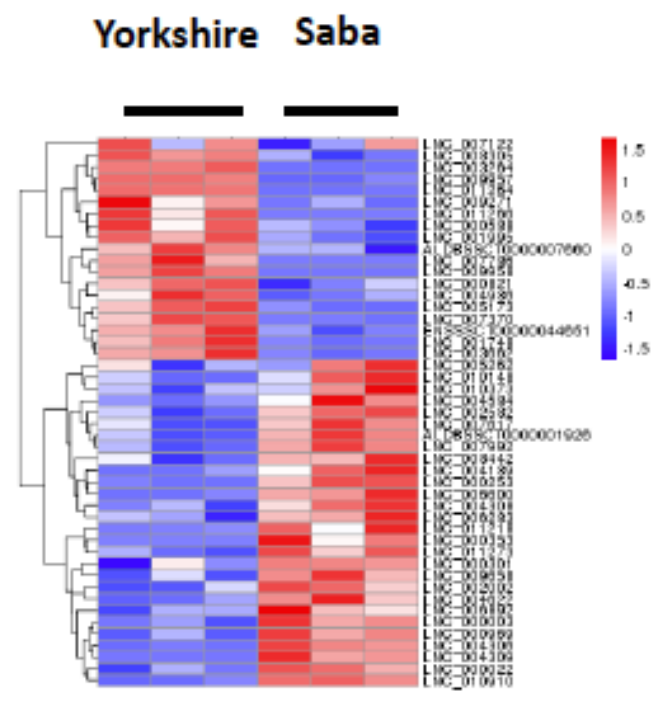

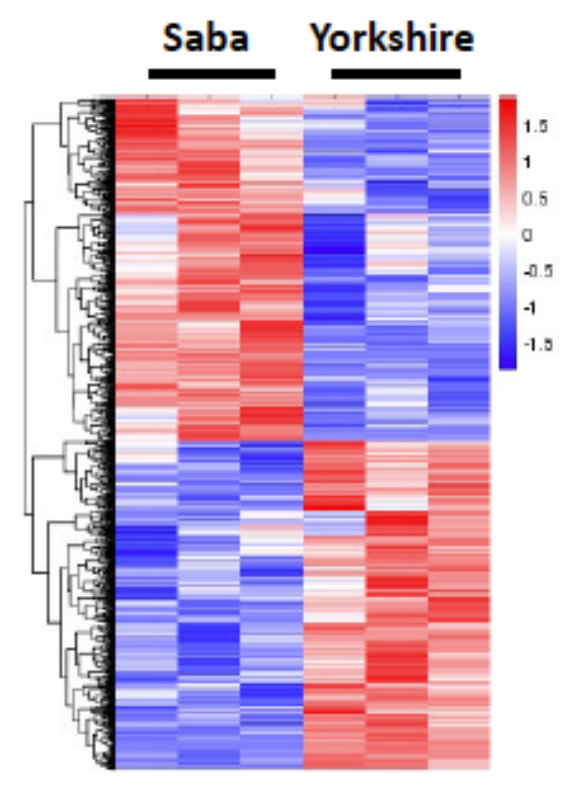

$\mathrm{D}$

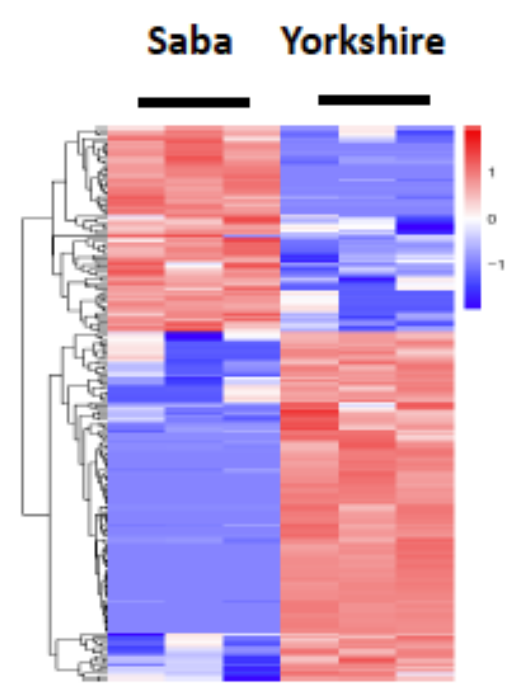

\section{Figure 3}

Heatmap of differentially expressed transcript of four RNAs. (A) DEmiRNAs; (B) DEmRNAs; (C) DElncRNAs and (D) DEmiRNAs. Horizontal axis represented the samples, and vertical axis represented differentially expressed RNAs. Red represented up-regulated RNAs, and blue represented down-regulated RNAs. 


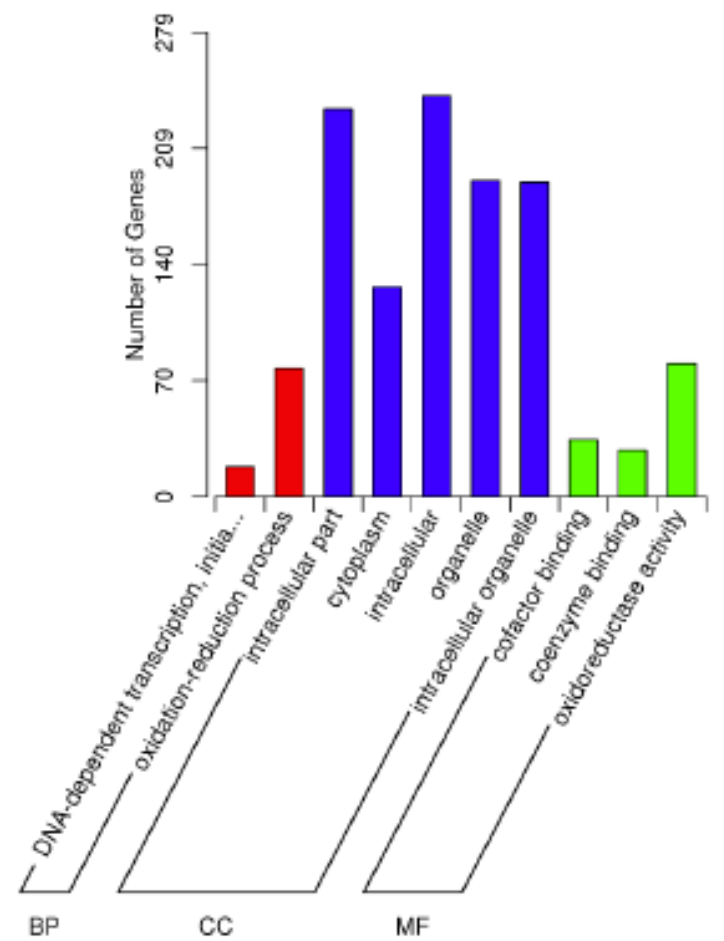

B

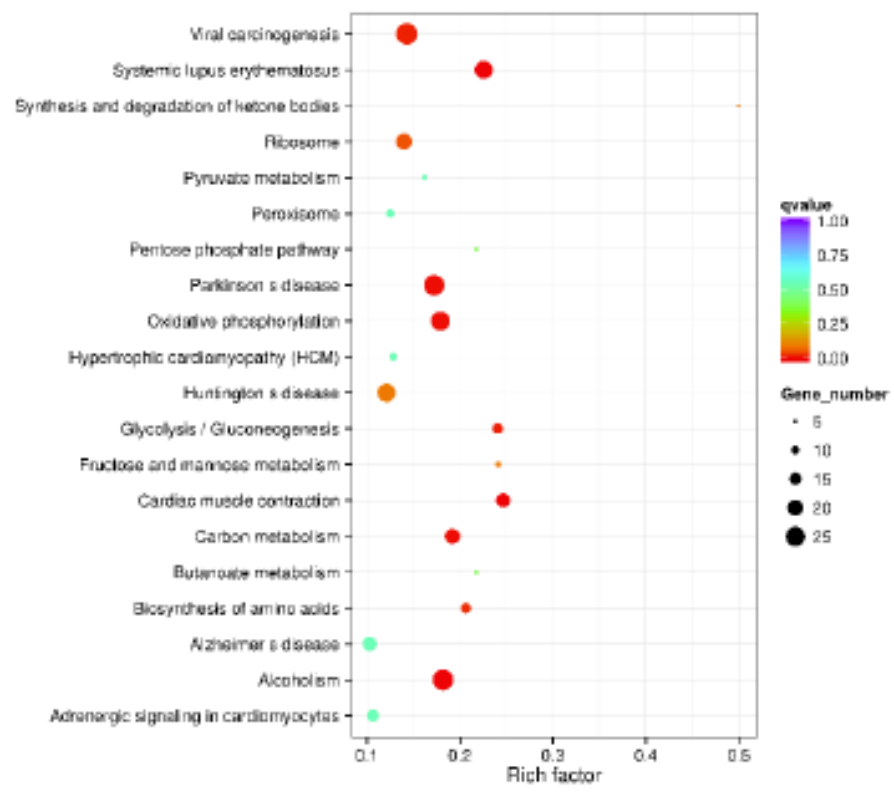

\section{Figure 4}

Functional enrichment analysis of differentially expressed mRNAs. (A) GO enrichment analysis. Two BPs, five CCs and three MFs were significantly enriched. (B) KEGG enrichment analysis. Twenty KEGG pathways were significantly enriched. GO, gene ontology; BPs, biological processes; CCs, cellular components; MFs, molecular functions; KEGG, Kyoto Encyclopedia of Genes and Genomes. 


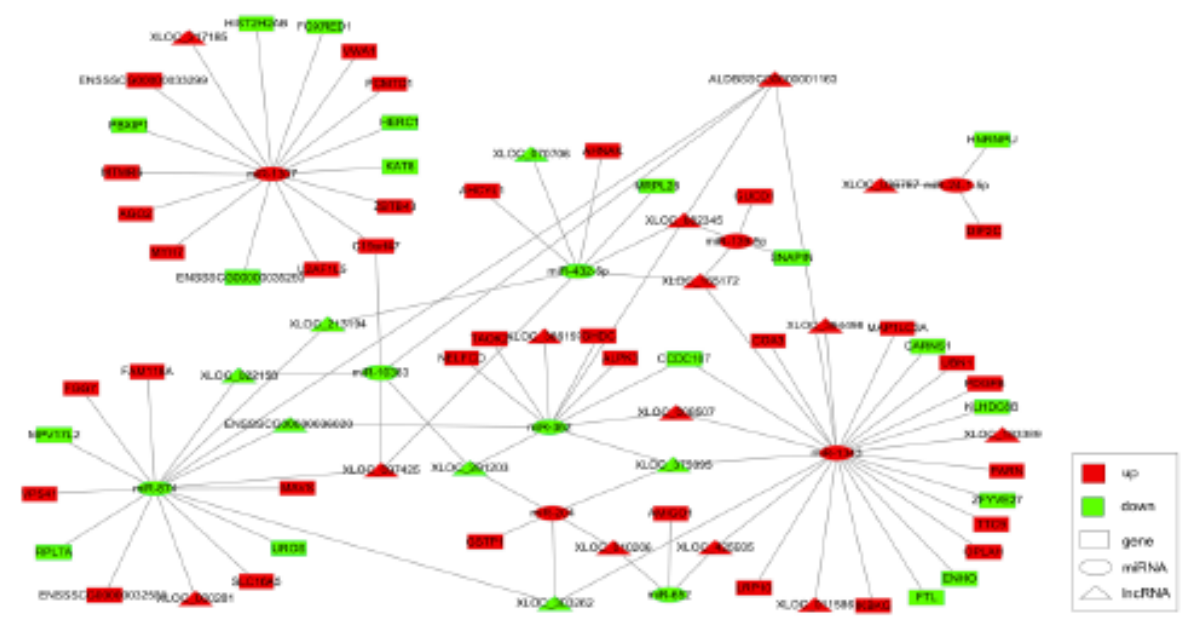

B

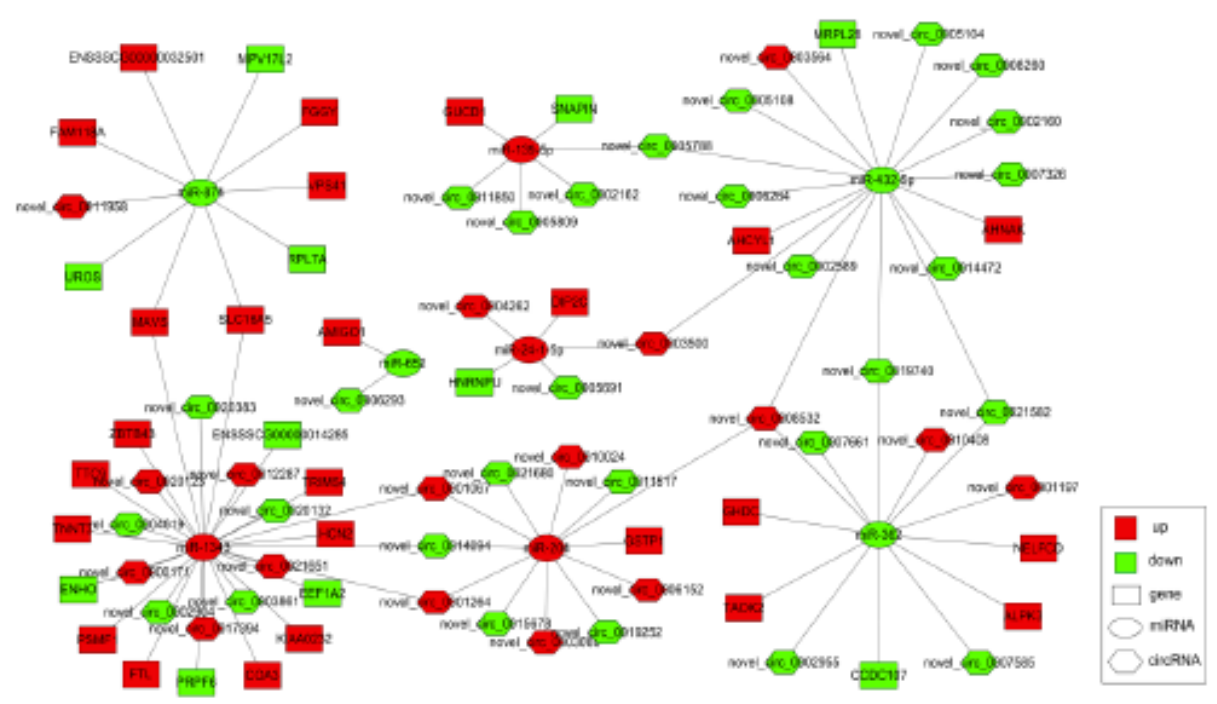

\section{Figure 5}

CeRNA network. On the basis of ceRNA theory, two ceRNAs were established. (A) IncRNA-miRNA-mRNA ceRNA network; (B) circRNA-miRNA-mRNA ceRNA network. Red represented up-regulated RNAs, and green represented down-regulated RNAs. Rectangle represented mRNA, and ellipse represented miRNA, and triangle represented IncRNA, and hexagon represented circRNA. MiR-1343 was identified to have more links in two ceRNA networks. ceRNA, competitive endogenous RNA. 

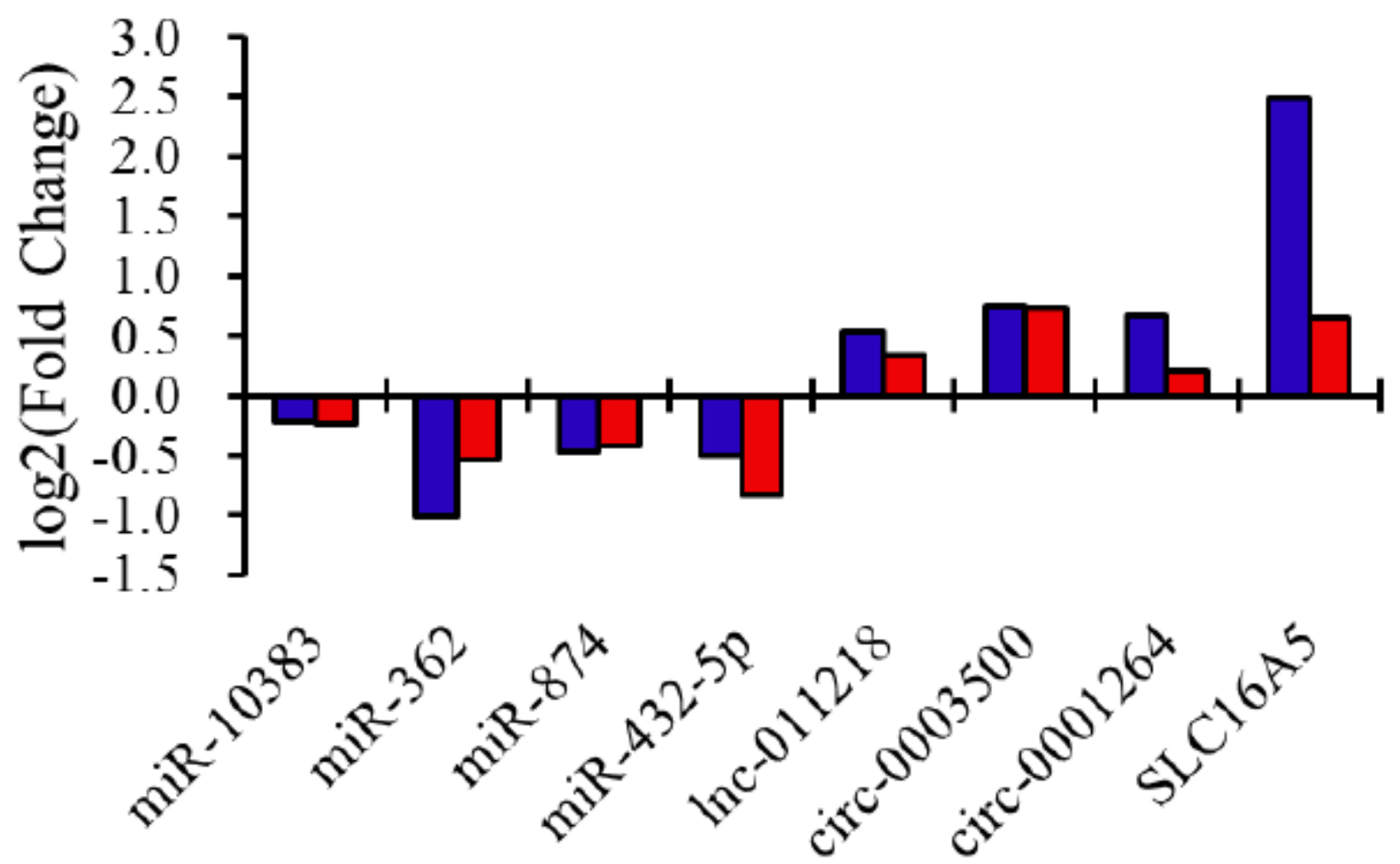

Figure 6

Validation of differentially expressed RNAs by qRT-PCR. Eight RNAs were randomly selected to validate the expression of DERNAs, and the results obtained from two methods were consistent.

\section{Supplementary Files}

This is a list of supplementary files associated with this preprint. Click to download.

- Supplementary.zip

- ARRIVEGuidelinesChecklist.pdf 